\title{
Native SAD data collection using microbeam at NSLSII
}

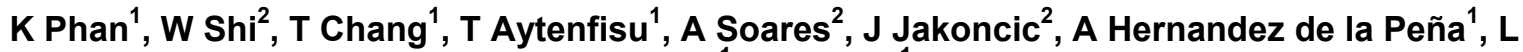 \\ Amzel ${ }^{1}$, S Gabelli ${ }^{1}$ \\ ${ }^{1}$ Johns Hopkins University School of Medicine, Baltimore, USA, ${ }^{2}$ Brookhaven National Laboratory, \\ NY, USA \\ kimphan15@augustana.edu
}

\begin{abstract}
Ab-initio single-wavelength anomalous dispersion (SAD) determination of macromolecular structures requires precise measurement of anomalous signal from atoms present in the sample such as $\mathrm{S}, \mathrm{Mn}, \mathrm{P}$ or introduced as heavy atoms. Today, availability of synchrotron microbeams and tunability at low-energy has increased the accessibility for measuring those data sets.
\end{abstract}

We described a native-SAD experiment collecting multiple data sets from a single crystal at an energy of $6550 \mathrm{eV}$ $\left(1.89 \AA\right.$ ) by offsetting the starting angle by $45^{\circ}$ with a beam size of 5 (v) x 7 (h) and an attenuated flux of $5.85 \mathrm{e} 10$ $\mathrm{ph} / \mathrm{s}$ at the NSLS-II FMX beamline.

We used chymotrypsinogen crystals with approximate dimensions of $20 \times 30 \times 140 \mu \mathrm{m}$, which contain one molecule of 245 amino acids in one asymmetric unit. Hence the asymmetric unit contains 10 cysteine residues in disulfide bond pairs and 2 methionine residues for a total 12 sulfur anomalous scattering atoms which result in a calculated Bijvoet fraction of $1.08 \%$. Five $360^{\circ}$ data sets were collected per crystal. Specifically, the data was collected using exposures of 0.02 seconds per $0.2^{\circ}$ for $360^{\circ}$ in a vector of about $138 \mu \mathrm{m}$ in length on an EIGER $16 \mathrm{M}$ detector. For the consecutive data sets, the crystal was rotated by $45^{\circ}$ and data was collected again with the same vector until the crystal showed apparent radiation damage. The average radiation dose estimated by RADDOSE was 1.63 MGy per data set.

The data was merged with BLEND and the structure was determined ab-initio using CRANK, the automated package for structure solution via experimental phasing. At each step, the specific programs used are SHELXC/D for the heavy atom location, Solomon for density modification and hand determination, PARROT for density modification, Buccaneer for automated model building and Refmac5 for refinement.

The work reported here with multiple data collections from the same crystal with low dose is an alternative to merging data from multiple crystals to obtain highly redundant data sets while balancing maximum signal and minimal absorption.

Acta Cryst. (2020). A76, a58 